\title{
Assessment of reporting quality of abstracts of systematic reviews with meta- analysis using PRISMA-A and discordance in assessments between raters without prior experience
}

\author{
Katarina Maticic ${ }^{1 \dagger}$, Marina Krnic Martinic ${ }^{2 \dagger}$ and Livia Puljak ${ }^{3 *}$ (D)
}

\begin{abstract}
Background: Reporting quality of systematic reviews' (SRs) abstracts is important because this is often the only information about a study that readers have. The aim of this study was to assess adherence of SR abstracts in the field of anesthesiology with the reporting checklist PRISMA extension for Abstracts (PRISMA-A) and to analyze to what extent will the use of PRISMA-A yield concordant ratings in two raters without prior experience with the checklist.

Methods: We analyzed reporting quality of SRs with meta-analysis of randomized controlled trials of interventions published in the field of anesthesiology from 2012 to 2016 by using 12-item PRISMA-A checklist. After calibration exercise, two authors without prior experience with PRISMA-A scored the abstracts. Primary outcome was median adherence to PRISMA-A checklist. Secondary outcome was adherence to individual items of the checklist. We analyzed whether there was improvement in reporting of SR abstracts over time. Additionally, we analyzed discrepancies between the two raters in scoring individual PRISMA-A items.

Results: Our search yielded 318 results, of which we included 244 SRs. Median adherence to PRISMA-A checklist was $42 \%$ (5 items of 12). The majority of analyzed SR abstracts $(N=148,61 \%)$ had a total adherence score under $50 \%$, and not a single one had adherence above $75 \%$. Adherence to individual items was very variable, ranging from 0\% for reporting SR funding, to $97 \%$ for interpreting SR findings. Overall adherence to PRISMA-A did not change over the analyzed 5 years before and after publication of PRISMA-A in 2013. Even after calibration exercise, discrepancies between the two raters were found in 275 (9.3\%) out of 2928 analyzed PRISMA-A items. Cohen's Kappa was 0.807 . In the item about the description of effect there were discrepancies in $59 \%$ of the abstracts between the raters.

Conclusion: Reporting quality of systematic review abstracts in the field of anesthesiology is suboptimal, and did not improve after publication of PRISMA-A checklist in 2013. We need stricter adherence to reporting checklists by authors, editors and peer-reviewers, and interventions that will help those stakeholders to improve reporting of systematic reviews. Some items of PRISMA-A checklist are difficult to score.
\end{abstract}

Keywords: Abstract, Systematic review, Reporting, PRISMA

\footnotetext{
*Correspondence: livia.puljak@gmail.com; livia.puljak@unicath.hr

† Katarina Maticic and Marina Krnic Martinic contributed equally to this work.

${ }^{3}$ Center for Evidence-Based Medicine and Health Care, Catholic University of

Croatia, llica 242, 10000 Zagreb, Croatia

Full list of author information is available at the end of the article
}

(c) The Author(s). 2019 Open Access This article is distributed under the terms of the Creative Commons Attribution 4.0 International License (http://creativecommons.org/licenses/by/4.0/) which permits unrestricted use, distribution, and reproduction in any medium, provided you give appropriate credit to the original author(s) and the source, provide a link to the Creative Commons license, and indicate if changes were made. The Creative Commons Public Domain Dedication waiver (http://creativecommons.org/publicdomain/zero/1.0/) applies to the data made available in this article, unless otherwise stated. 


\section{Background}

Usability and transparency of literature depends on its reporting quality. For this reason, many reporting checklist have been developed, for various types of studies, including study abstracts, facilitated by the EQUATOR Network (Enhancing the Quality and Transparency Of health Research) [1, 2]. Reporting quality of abstracts is particularly important because readers will often have access only to an abstract of a manuscript and even if they have access to the full text they will often decide about reading the full text depending on the information available in the abstract. Furthermore, manuscript published in languages other than English may have only abstract available in English, and then the abstract becomes the only source of information that is available to readers [3].

Systematic reviews (SRs) are considered to be the gold standard of evidence synthesis [4], and as such, quality of their reporting is of paramount importance. In 2013, the Preferred Reporting Items for Systematic Reviews and Meta-Analyses (PRISMA) extension for abstracts (PRISMA-A) checklist was published with the aim of improving reporting of SR abstracts [3]. To our best knowledge, only a few studies so far have analyzed reporting quality of SR abstracts according to the PRISMA-A that were published as full-text manuscripts in scholarly journals [5-8]. However, they were focused either on general topics $[6,8]$, or very specific topics i.e. depression screening tool [5] or Iranian SRs [7] and two of them included abstracts that were published before publication of PRISMA-A [7, 8].

Considering the importance of abstracts' reporting, there was minimal effort so far invested in analyzing whether SR authors adhere to the PRISMA-A. Since pain management is considered fundamental human right [9], it could be argued that importance of proper reporting in the field of anesthesiology and pain is extremely important for both patients and health care workers. Therefore, the aim of this study was to analyze reporting quality of SRs with meta-analysis of randomized controlled trials (RCTs) of interventions published in the field of anesthesiology from 2012 to 2016, to see whether there was a temporal trend towards improvement of abstracts' reporting quality after introduction of the PRISMA-A checklist.

\section{Methods}

\section{Ethics}

This study analyzed data from published studies; no patient data were included.

\section{Definitions}

We defined SR as a study with a clear research question, systematic methods to identify, select and appraise the literature, where authors extracted and analysed/synthesized data from included studies [10]. Studies were not considered "systematic" if they had only one author or that searched only one database [11].

\section{Inclusion criteria}

We included SRs/meta-analyses of RCTs about interventions published from 2012 to 2016 in all journals within the JCR category Anesthesiology, based on the Journal Citation Reports (JCR) impact factor for year 2016.

\section{Study search and screening}

We searched PubMed using a filter for meta-analysis, date (years 2012-2016) and journal name. We exported search results, and screened eligibility of the abstracts. One author screened the abstracts and the second author verified the screening results.

\section{Exclusion criteria}

We excluded diagnostic SRs, empty SRs (i.e. did not include a single study), withdrawn SRs and overviews of systematic reviews. If the SR included both randomized and non-randomized studies, it was excluded.

\section{Abstracts' reporting quality}

Two authors independently analyzed reporting quality of included SR abstracts against the 12 items of the (PRISMA-A). We reviewed in detail original publication that described PRISMA-A [3] and developed a coding manual. We performed a calibration exercise by two authors on a sample of ten abstracts. Both raters of abstracts have a biomedical degree, with no previous experience with PRISMA-A. We scored individual items of PRISMA-A as 'yes' or 'no', according to the coding manual. We analyzed percentage of discrepancies between the two independent authors for each item of the PRISMA-A. All discrepancies between the two authors were resolved in the consensus phase via discussion and finalized data sheet with scores was analyzed. For items with initial discrepant opinion, the final adherence status was determined by the agreed result in the consensus phase.

\section{Outcomes}

Primary outcome was total median adherence to PRISMA-A. Secondary outcome was adherence to individual items of PRISMA-A.

\section{Data analysis}

We made descriptive statistics using frequencies and percentages for each analyzed item. Adherence to PRISMA-A checklist for each item was shown with median and interquartile range (IQR). For analysis we used MedCalc software (MedCalc Corp., Mariakerke, Belgium). 


\section{Results}

Our search yielded 318 results, of which we included 244 SRs. We excluded 74 records because they were not systematic reviews, did not analyze interventions or did not include meta-analysis.

\section{Primary outcome: median adherence to PRISMA-A}

Median adherence to PRISMA-A in the analyzed 244 SRs was $42 \%$ (IQR 42 to $50 \%$ ), i.e. 5 PRISMA-A items (IQR 5 to 6 items). The majority of analyzed SR abstracts $(N=148,61 \%)$ had a total adherence score under $50 \%$. Only 4SRs $(1.6 \%)$ had a total adherence score between 60 and $75 \%$ and not a single SR had total adherence to PRISMA-A that was above $75 \%$. We did not see any improvement in the adherence to PRISMA-A over the analyzed 5 years; overall median adherence to all items of PRISMA-A in four of the five analyzed years was $42 \%$, while in 2013 median adherence was $46 \%$. Median adherence to individual items of PRISMA-A is shown in (Table 1), indicating that these medians did not change over time.

\section{Secondary outcome: proportion of SR abstracts that adhere to individual items of PRISMA-A}

The lowest adherence with individual items of PRISMA-A was found for item 11 (funding), as neither one of the analyzed SR abstract indicated source of funding. Only two SR abstracts adhered to the item 12 (registration). Adherence of five items was above $80 \%$; whereas adherence above $90 \%$ was found for only two items: 1 (title) and 10 (interpretation) (Table 2).

\section{Discrepancies between the raters}

After all abstracts were checked against PRISMA-A by two authors individually, we analyzed discrepancies between the two authors. Even after calibration exercise, discrepancies were found in 275 (9.3\%) out of 2928 analyzed PRISMA-A items. On average, there were 1.1 discrepant assessments per abstract. Cohen's Kappa was 0.807. Number of discrepancies in each item is shown in Table 1. There were no discrepancies at all in items 1 (title), 4 (information sources), 11 (funding) and 12 (registration). Few discrepancies were observed for items

Table 1 Median adherence to individual items of reporting checklist PRISMA for Abstracts over time

\begin{tabular}{lllllllllllll}
\hline Year & \multicolumn{1}{l}{ PRISMA-A item } \\
\cline { 2 - 13 } & 1 & 2 & 3 & 4 & 5 & 6 & 7 & 8 & 9 & 10 & 11 & 12 \\
\hline 2012 & 1 & 1 & 0 & 0 & 0 & 0 & 1 & 1 & 0 & 1 & 0 & 0 \\
2013 & 1 & 1 & 0 & 0 & 0 & 0 & 1 & 1 & 0 & 1 & 0 & 0 \\
2014 & 1 & 1 & 0 & 0 & 0 & 0 & 1 & 1 & 0 & 1 & 0 & 0 \\
2015 & 1 & 1 & 0 & 0 & 0 & 0 & 1 & 1 & 0 & 1 & 0 & 0 \\
2016 & 1 & 1 & 0 & 0 & 0 & 0 & 1 & 1 & 0 & 1 & 0 & 0 \\
\hline
\end{tabular}

3 (eligibility criteria), 7 (synthesis of results) and 10 (interpretation). For three items discrepancies were observed in $6-11 \%$ of abstracts: items 5 (risk of bias), 7 (synthesis of results) and 9 (strengths and limitations of evidence). For item 2 (objectives) and particularly for item 8 (description of effect) we found high number of discrepancies between the two raters (Table 2).

\section{Discussion}

We found that adherence of abstracts indexed as systematic reviews and meta-analysis to PRISMA-A reporting checklist in the field of anesthesiology was suboptimal, as median adherence to the checklist was $42 \%$ and there was no improvement in abstract reporting over the analyzed 5 years.

Very few studies were done so far on this topic. We found four published manuscripts in peer-reviewed literature that compared reporting in SR abstracts with PRISMA-A. One of them, published by Hopewell et al. in 2015, analyzed reporting of 103 SR abstracts presented at nine leading international conferences in 2010 and subsequently published in journals [8]. Since their sample of abstracts were published before publication of PRISMA extension for Abstracts in 2013 [3], the study of Hopewell at al. can be used to asses pre-PRISMA-A reporting status of abstracts. The authors themselves concluded their manuscript with the following words [quote]: "Our study will provide important baseline information, before publication of PRISMA for Abstracts guidelines, against which future impact can be measured." [8].

However, Hopewell et al. did not use the 12 original items of the PRISMA-A as they are presented in the checklist. Instead, they split some of the items into more items. For example, item 4 about information sources was split into two items, one for key databases searched and one for search dates. Item 8 about description of effect was split into two items, one for benefit and one for harms [8]. Therefore, our results are not directly comparable for all PRISMA-A items. Results that are completely matching in our study and study of Hopewell et al. is that abstracts presented at nine major international biomedical conferences in 2010 also did not include information about SR funding and registration [8], and this was not different in years 2012-2016 in journals from the field of anesthesiology, as our study shows. Only two items that had adherence with PRISMA-A above $80 \%$ in the study of Hopewell et al. were item 1 (title) with $89 \%$ of adherence and sub-item 4 (number and type of included studies) with $81 \%$ of adherence [8]. In our study we had five PRISMA-A items with adherence above $80 \%$, so this could be considered an improvement following the introduction of PRISMA-A. 
Table 2 Adherence to individual items of PRISMA-A in analyzed systematic reviews and frequency of discrepancies between two authors for each item

\begin{tabular}{|c|c|c|c|c|c|}
\hline No. & PRISMA-A item & Description & $\begin{array}{l}\text { Adherent, } \\
N(\%)^{\mathrm{a}}\end{array}$ & $\begin{array}{l}\text { Non-adherent, } \\
N(\%)^{\mathrm{a}}\end{array}$ & $\begin{array}{l}\text { Discrepancies in rating } \\
\text { between raters, } N(\%)^{\mathrm{a}}\end{array}$ \\
\hline 1 & Title & $\begin{array}{l}\text { Identify the report as a systematic review, } \\
\text { meta-analysis, or both }\end{array}$ & $234(95.9)$ & $10(4.1)$ & $0(0)$ \\
\hline 2 & Objectives & $\begin{array}{l}\text { The research question including components } \\
\text { such as participants, interventions, comparators, } \\
\text { and outcomes }\end{array}$ & $216(88.5)$ & $28(11.5)$ & $46(19)$ \\
\hline 3 & Eligibility criteria & $\begin{array}{l}\text { Study and report characteristics used as criteria } \\
\text { for inclusion }\end{array}$ & $17(7)$ & $227(93)$ & $3(1.2)$ \\
\hline 4 & Information sources & Key databases searched and search dates & $71(29.1)$ & $173(70.9)$ & $0(0)$ \\
\hline 5 & Risk of bias & Methods of assessing risk of bias & $12(4.9)$ & $232(95.1)$ & $16(6.6)$ \\
\hline 6 & Included studies & $\begin{array}{l}\text { Number and type of included studies and } \\
\text { participants and relevant characteristics of studies }\end{array}$ & $29(11.9)$ & $215(88.1)$ & $28(11)$ \\
\hline 7 & Synthesis of results & $\begin{array}{l}\text { Results for main outcomes (benefits and harms), } \\
\text { preferably indicating the number of studies and } \\
\text { participants for each. If meta-analysis was done, } \\
\text { include summary measures and confidence intervals }\end{array}$ & $202(82.8)$ & $42(17.2)$ & $9(3.7)$ \\
\hline 8 & Description of the effect & $\begin{array}{l}\text { Direction of the effect (i.e. which group is } \\
\text { favoured) and size of the effect in terms } \\
\text { meaningful to clinicians and patients }\end{array}$ & $210(86.1)$ & $34(13.9)$ & $143(59)$ \\
\hline 9 & $\begin{array}{l}\text { Strengths and limitations of } \\
\text { evidence }\end{array}$ & $\begin{array}{l}\text { Brief summary of strengths and limitations of } \\
\text { evidence (e.g. inconsistency, imprecision, } \\
\text { indirectness, or risk of bias, other supporting or } \\
\text { conflicting evidence) }\end{array}$ & $48(19.7)$ & $196(80.3)$ & $23(9.4)$ \\
\hline 10 & Interpretation & $\begin{array}{l}\text { General interpretation of the results and important } \\
\text { implications }\end{array}$ & $236(96.7)$ & $8(3.3)$ & $7(2.9)$ \\
\hline 11 & Funding & Primary source of funding for the review & $0(0)$ & $244(100)$ & $0(0)$ \\
\hline 12 & Registration & Registration number and registry name & $2(0.8)$ & $242(99.2)$ & $0(0)$ \\
\hline
\end{tabular}

aPercentages calculated from the total number of analyzed abstracts: 244

Rice et al. analyzed reporting of 21 SRs with meta-analyses using adapted PRISMA-A checklist; the authors adapted the PRISMA-A because they analyzed only studies about depression screening tool accuracy, and therefore these SRs dit not analyze interventions but diagnostic test accuracy (DTA) [5]. While their results indicated that quality and completeness of reporting in the analyzed SRs were suboptimal [5], we have to emphasize that they analyzed SRs published from 2007 to 2016. Beller et al. published PRISMA-A checklist in April of 2013 [3], and if we assume some lag time needed for raising awareness of the PRISMA-A, it would be reasonable to expect that manuscripts published in 2014 or 2015 could be expected to adhere to the PRISMA-A checklist.

Rice et al. have presented detailed analysis of adapted PRISMA-A for each of their 21 included studies in their manuscript. By analyzing their data, it can be seen that even if we limit post-PRISMA-A SR abstracts to year 2015 and afterwards, this would include 5 of their 21 SRs, and median total percent of adherence to the adapted PRISMA-a was 29\% before 2015 and 36\% in SR abstracts published in 2015 and 2016, i.e. post-PRISMA-a. Even if we take two SRs published in 2014 as post-PRISMA-A cohort, the median total adherence is still 36\% [5].
Rice et al. split item 3 and 4 into two sub-items. Their overall results indicated that items 2 (objectives), $3 \mathrm{~b}$ (report characteristics), 11 (funding) and 12 (registration) were fulfilled in virtually no abstracts. The highest adherence in their sample of abstracts was found for reporing item 1 (title) and 10 (interpretation) [5], which was in agreement with our results. Some results were strikingly different; for example in the study of Rice et al. item 2 (objectives) was reported in $0 \%$ of abstracts, and in our study in $89 \%$ of abstracts, but this could have been due to the fact that they analyzed diagnostic test accuracy (DTA) studies and their adapted item 2 asked for "the research question including components such as participants, index test, reference standard and outcomes" [5].

Bigna et al. used PRISMA-A checklist to analyze quality of 204 abstracts of SRs with meta-analysis of RCTs published in high-impact general medicine journals in 2012, 2014 and 2015. They reported that the mean number of items reported was 7.2 in 2012, 6.8 in 2014 and 7.5 in 2015 [6]. In our sample the median number of reported numbers was 5 in all analyzed years. Bigna et al. used original 12 PRISMA-A items without adaptations. In their methods the authors indicated that they 
included "all systematic reviews" in the specified time frame, without specifying whether they included only SRs of interventions, and how did they score SRs of DTA and other types of SRs if they had such reviews in their sample. Bigna et al. concluded that the reporting quality of analyzed SR abstracts did not improve in 2014 and only slightly improved in 2015; they also found that reporting was better in abstracts structured with 8 headings and abstracts with word count under 300 words [6].

This finding that the reporting was better in shorter abstract is highly relevant, because it may be intuitive to consider that the quality of reporting may be hindered with restrictive word counts. The findings of Bigna et al. refer to word count under 300 words [6], but some journals, for example, require abstracts limited to 200 words, and in those circumstances it may be difficult to report all key domains suggested by the PRISMA-A. Journal editors should reconsider using very restrictive word counts in abstracts to enable proper reporting. As the authors of the PRISMA-A indicated, abstracts are not supposed to replace full-text manuscript, but for time-pressed readers or those without access to full-text reports, it is important that abstract contains relevant information [3].

Bigna et al. did not present detailed results for all analyzed abstracts in terms of adherence to individual items of PRISMA-A; instead showed those results separately for three cohorts of abstracts published in each year in Table 3 [6]. From this table one can see that the results in terms of adherence to individual PRISMA-A items were very different in some items in different years, and similar in other items; there was no uniform trend of improvement in adherence in all PRISMA-A items in analyzed abstracts over the 3 years. Results that stand out are those for items 11 (funding) with overall adherence across 3 years of $28 \%$ and 12 (registration) with a total adherence of 9.3\% [6]. Adherence to those two items was much better compared to other studies, including ours.

In 2017, Kazerani et al. reported their analysis of abstracts of Iranian SRs and meta-analyses indexed in Web of Science (WoS) and Scopus published from 2003 to 2012 by using PRISMA-A [7]. However, PRISMA-A was published until 2013 [3], so the authors of the abstracts analyzed by Kazerani et al. [7] did not yet have a chance to adhere to the reporting checklist that was not available at the time the SR was published. Kazerani et al. showed that the reporting quality of 293 analyzed SR abstracts was suboptimal, with the highest adherence on item 1 (title), 2 (objectives) and 7 (synthesis of results), which was above $80 \%$ for all three. The lowest adherence was described for items 3 (eligibility criteria), 5 (risk of bias), 6 (included studies), 9 (strengths and limitations) and 12 (registration) with adherence under $6 \%$ for all those items [7].

Our data also imply that rating adherence to reporting checklists should be done by at least two raters because we had high discrepancies in few checklist items; for one item - description of the effect - we had discrepancies between two raters in as many as $59 \%$ of analyzed abstracts. On the contrary, for four items we did not have a single discrepancy, which indicates that some items of PRISMA-A are straightforward for scoring and not ambiguous at all. These four items with no discrepancies address reporting of title, information sources, funding and protocol registration. We had overall high number of discrepancies between the two raters, and the overall inter-rater agreement was high, and the majority of discrepancies were driven by differences in scoring of the two items -item \#2 about objectives and item 8 about description of effect. Raters in this study were first-time raters with no prior experience with PRISMA-A, and this could have contributed to differences, but as our results indicate, discrepancies were not evenly distributed between different PRISMA-A items, which indicates that some items are more difficult to score uniformly across raters.

In the four publications that we found that used PRISMA-A for analyzing reporting of SR abstracts [6,7], two reported that two authors analyzed abstracts independently $[5,6]$, one vaguely indicated that data extraction "was carried out by teams of assessors working in pairs" [8], and one did not report the method for analyzing the abstracts in terms of number of raters [7]. Only one of the four manuscripts reported overall inter-rater agreement; indicating that the agreement between reviewers on all PRISMA-A items was high $($ Kappa $=0.79)$ [6]. Without more detailed information about potential difficulties with scoring individual PRISMA-A items, we cannot know whether other authors had problems with the same items as we did in this study.

Our study is one of a number of methodological studies in the field of anesthesiology, showing deficiencies in the published literature and suboptimal reporting. We have recently showed that authors of RCTs published in top anesthesiology journals do not report using SRs to inform their study design [12]. We also found that RCT abstracts presented in four consecutive World Congresses on Pain are not properly reported and not necessarily dependable sources of information, as there are frequently discrepant results between RCT presented at a conference and published subsequently in a peer-reviewed journal [13]. As Eccleston et al. indicated in 2010, there are tools that can help authors improve quality and reporting of SRs [14]. While validity of a SR may be inherently linked with the study design and time-consuming to improve after the study is completed, improving quality of reporting of SRs should not be so demanding.

Findings of our study indicate that we need interventions aimed towards authors, editors and peer-reviewers, which will improve reporting of abstracts of systematic reviews. Authors may not be aware of reporting guidelines, but 
editors and peer-reviewers are gate-keepers that can insist on reporting standards as prerequisites for publication. Many journals have endorsed PRISMA statement, but PRISMA-A is extension, and therefore instructions for authors should explicitly mention not only PRISMA, but also its extensions, so that authors can comply with them.

It could be argued that suboptimal compliance with a reporting checklist for abstract is due to inadequate peer-review, and that peer-reviewers should pay more attention to the quality of reporting. However, reviewers themselves may not be aware of the existence of the relevant reporting checklist for the type of manuscript they are reviewing, and journals will rarely specifically instruct reviewers to check a manuscript against a reporting checklist. Editors could contribute to enhanced peer-review in terms of reporting quality by asking reviewers explicitly to provide feedback about the compliance of a manuscript with relevant checklists that will address both abstract and the full text.

Furthermore, it could be argued that suboptimal reporting of the abstract can be an indicator of suboptimal reporting of the entire manuscript, but we did not study this potential association in our manuscript. The original PRISMA statement was published in 2009 [15], and the PRISMA-A extension was published 4 years later. It is possible that the authors are aware of the PRISMA and use it for reporting the full manuscript, but not aware of the existence of PRISMA-A.

In this study we did not analyze whether journals mention PRISMA-A in their instructions for authors, because we analyzed manuscripts published over 5 years, and there is also lag between submission and publication, and instructions for authors may change and evolve. For this reason, current state of instructions for authors would not be informative for those past publications, but it is something that editors should consider to include explicitly in their instructions if they want better reporting.

Additionally, editors can use tools such as Penelope to help authors evaluate their manuscripts before submission, or after submission, to help them achieve better adherence with reporting guidelines [16]. Penelope or a similar information technology tool could be customized to help authors and editors improve reporting in systematic reviews.

\section{Conclusions}

Reporting quality of analyzed abstracts of systematic reviews of interventions with meta-analysis in the field of anesthesiology was suboptimal, and it did not improve after publication of PRISMA-A checklist in 2013. We need stricter adherence to reporting checklists by authors, editors and peer-reviewers, and interventions that will help those stakeholders to improve reporting of systematic reviews.

\section{Abbreviations}

DTA: Diagnostic test accuracy; EQUATOR Network: Enhancing the Quality and Transparency Of health Research; IQR: Interquartile range;

PRISMA: Preferred Reporting Items for Systematic Reviews and Meta-

Analyses; PRISMA-A: Preferred Reporting Items for Systematic Reviews and Meta-Analyses extension for abstracts; RCT: Randomized controlled trial;

SR: Systematic reviews; WoS: Web of Science

\section{Acknowledgements}

This manuscript is based on a diploma thesis of Ms. Katarina Maticic; the thesis was written and defended in Croatian language.

\section{Funding}

No extramural funding.

\section{Availability of data and materials}

Analyzed data were publicly available information available in the published literature. The datasets used and/or analyzed during the current study are available from the corresponding author on reasonable request.

\section{Authors' contributions}

Study design: LP. Data collection, analysis and interpretation: KM; MKM, LP. Writing first draft of the manuscript: LP. Critical revisions of the manuscript: KM; MKM, LP. Approval of the final version of the manuscript to be submitted: KM; MKM, LP. Agree to be accountable for all aspects of the work: KM; MKM, LP.

Ethics approval and consent to participate

Not applicable; in this study we analyzed only published literature.

\section{Consent for publication}

Not applicable

\section{Competing interests}

Livia Puljak is a member of the editorial board (Section Editor) of the BMC Medical Research Methodology.

\section{Publisher's Note}

Springer Nature remains neutral with regard to jurisdictional claims in published maps and institutional affiliations.

\section{Author details}

${ }^{1}$ Pharmacy Mirna Jelic Hrgic, Brodski Stupnik, Croatia. ${ }^{2}$ Department of ENT, Head and Neck Surgery, University Hospital Center, Split, Croatia. ${ }^{3}$ Center for Evidence-Based Medicine and Health Care, Catholic University of Croatia, Ilica 242, 10000 Zagreb, Croatia.

Received: 25 October 2018 Accepted: 7 February 2019

Published online: 14 February 2019

\section{References}

1. EQUATOR Network Available at: http://www.equator-network.org/. Accessed 12 Feb 2019.

2. EQUATOR: Enhancing the QUAlity and Transparency Of health Research. Available at: http://www.equator-network.org/. Accessed 12 Feb 2019.

3. Beller EM, Glasziou PP, Altman DG, Hopewell S, Bastian H, Chalmers I, Gotzsche PC, Lasserson T, Tovey D. PRISMA for abstracts: reporting systematic reviews in journal and conference abstracts. PLoS Med. 2013; 10(4):e1001419.

4. Smith R: The Cochrane collaboration at 20 much has been achieved, but much remains to be done. Brit Med J. 2013; 347

5. Rice DB, Kloda LA, Shrier I, Thombs BD. Reporting quality in abstracts of meta-analyses of depression screening tool accuracy: a review of systematic reviews and meta-analyses. BMJ Open. 2016;6(11):e012867.

6. Bigna JJ, Um LN, Nansseu JR. A comparison of quality of abstracts of systematic reviews including meta-analysis of randomized controlled trials in high-impact general medicine journals before and after the publication of PRISMA extension for abstracts: a systematic review and meta-analysis. Syst Rev. 2016;5(1):174 
7. Kazerani M, Davoudian A, Zayeri F, Soori H. Assessing abstracts of Iranian systematic reviews and metaanalysis indexed in WOS and Scopus using PRISMA. Med J Islam Repub Iran. 2017;31:18.

8. Hopewell S, Boutron I, Altman DG, Ravaud P. Deficiencies in the publication and reporting of the results of systematic reviews presented at scientific medical conferences. J Clin Epidemiol. 2015.

9. Brennan F, Carr DB, Cousins M. Pain management: a fundamental human right. Anesth Analg. 2007;105(1):205-21.

10. CRD: Center for Reviews and Dissemination, University of York. Undertaking Systematic Reviews of Research on Effectiveness. Research report. CRD Report 2001, 4(2). York: NHS Center or Reviews and Dissemination; 2001.

11. Puljak $L$. If there is only one author or only one database was searched, a study should not be called a systematic review. J Clin Epidemiol. 2017.

12. Engelking A, Cavar M, Puljak L. The use of systematic reviews to justify anesthesiology trials: a meta-epidemiological study. Eur J Pain. 2018

13. Saric L, Vucic K, Dragicevic K, Vrdoljak M, Jakus D, Vuka I, Kadic AJ, Saldanha IJ, Puljak L. Comparison of conference abstracts and full-text publications of randomized controlled trials presented at four consecutive world congresses of pain: reporting quality and agreement of results. Eur J Pain. 2018. https://doi.org/10.1002/ejp.1289.

14. Eccleston C, Moore RA, Derry S, Bell RF, McQuay H. Improving the quality and reporting of systematic reviews. Eur J Pain. 2010;14(7):667-9.

15. Moher D, Liberati A, Tetzlaff J, Altman DG. Preferred reporting items for systematic reviews and meta-analyses: the PRISMA statement. Open Medicine. 2009;3(3):e123-30.

16. del Pozo Martin Y. BMJ Open trials Penelope. BMJ Open Blogs. Available at: http://blogs.bmj.com/bmjopen/2017/02/06/bmj-open-trials-penelope/. Accessed 12 Feb 2019

Ready to submit your research? Choose BMC and benefit from:

- fast, convenient online submission

- thorough peer review by experienced researchers in your field

- rapid publication on acceptance

- support for research data, including large and complex data types

- gold Open Access which fosters wider collaboration and increased citations

- maximum visibility for your research: over $100 \mathrm{M}$ website views per year

At $\mathrm{BMC}$, research is always in progress.

Learn more biomedcentral.com/submissions 